\title{
Propulsion Aspects of Unmanned and Manned Lunar Landings (*)
}

\author{
Dexis S. CAlRTON
}

Ricevuto il 19 Febbraio 1963

SUMMART. - Direct vertical deseent and descent from an intermediate lunar parking orhit are discussed. The difference in philosophy between langing a payload of instruments and of humans is considered in some detail. The "human return to eartl." problem is separated from the "mission success-failure" criteria.

Some anticipated performances are given in terms of payload ratio for various propulsion-cehicle vealing constants for both forms of descent.

Mammed descent from parking orbit is discussed with respect to the general problem of maximising the probability of human return. Minimum impulse, high safety and long low approaches are mentioned. In conclusion the problem of ahort during the final powered descent is considered for fixed and variable geometry vehicles.

Riassuxto. - Vengono discussi il rientro verticale diretto e l'atterraggio da un intermedio approdo lunare. Con le questioni di carattere tecnico viene esaminato l'annesso problema di carattere psicologico.

\section{IXTRODUCTIOX.}

Landing a payload gently upon the lumar surface is a proceeding of considerable complexity. A large part of the possible umreliability of such a process will depend upon the number of "reliability sensitive" components used in the whole landing process. The reliability of the whole process will depend both upon the number of separate steps involved, and upon the product reliability of the steps. When plamming

(*) Nota presentata al $2^{\circ}$ Congresso Internazionale Tecnico scientifieo dello Spazio. Roma, 19-23 Giugno 1962. 
to land instruments it is possible to demand a high reliability for adeh component and step -- or to chplicate where a high reliability does not appear otherwise economically obtainable. The overall system is one which, nevertheless, may still only have an anticipated suceess of five or six per ten "potential launches". No doubt in use mueh could be done to find the soures of unreliability in the lunar landing phase and so help to raise the odds against failure.

Consideration at this point will indicate that when a human is carried, a very different situation exists. The possibility of failure of a most complex single stream system inevitably depends upon the reliability of the processes within. At this moment it appears most improbable that a tested system of high enough demonstratable reliability can be achievert for a single stream. In consequence it becomes necessary to consider as completely sepalate the likelihoor of mission suceess, and the likelihood of human return. It has in fact now become essential to require as part of the overall system a "take me home safely" mechanism, which can be called upon to to do the magic campet acet at any point through the mission.

\section{ITSSAR APPROACHES.}

The landing velicele is in an umpowered orbit about the moon. There are orbits of two distinct sorts: those whose altitude above the lumar surface is always positive, and those wherein it is not. In consequence. two extreme cases will be considered here.

First, the powereel landing which starts in vertical plane. Secondly, the powered landing from a circular orbit.

\section{Vertical Descent Laxniva Techigle.}

\section{Unmanned.}

An early study of this mode of landing was carried out by Carton (1) which specifically considered the landing of instruments. Figure 1. baser on that reference shows the details of such an approach. It has become apparent since then that the landing pieture will depend to some extent on how great an imparet the payload could be planned to survive $\left.{ }^{2}\right)$. The plans from the L.S.A. all for increased control of rescent and touch down. 
As far as propulsion is concerned the main problem areas are:

(1) the selection of propellant type;

(2) selection of operating parameters to give a maximum landed load of instruments from a given (lunar) orbiting vehicle.

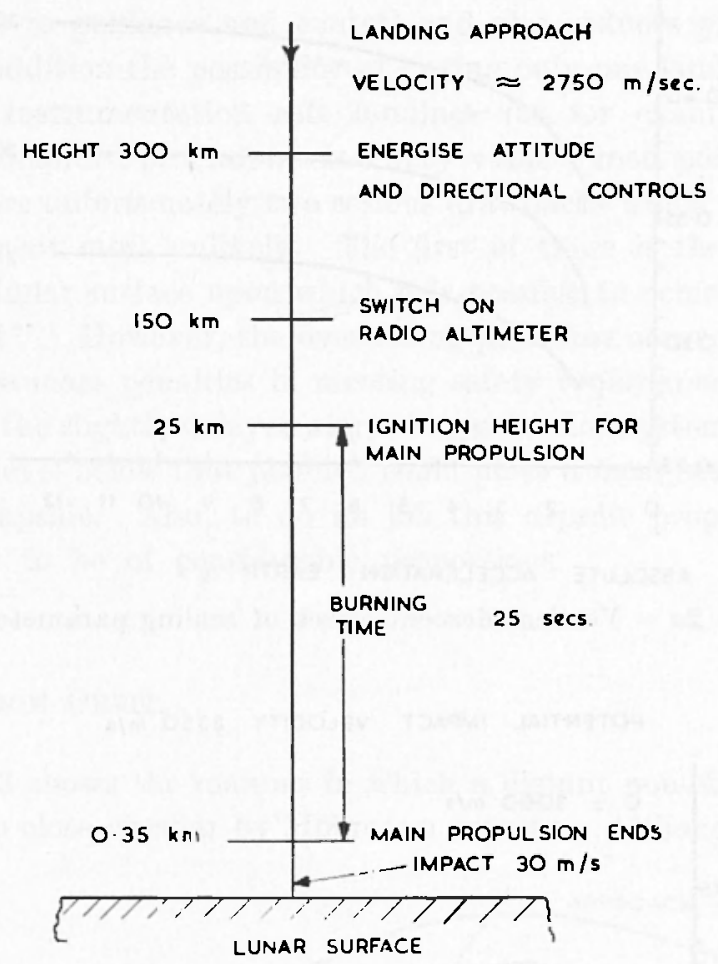

Fig. 1 - Vertical descent with "Hard Impact".

The general trend in propellant selection appears to be to use a solid propellant first, as it is more quickly available. Then to replace this with " storable" liquids at some intermediate point because a " thrust variability on demand" requirement is being put forward. Also a better payload achievement for stored liquid vis-a-vis solids after a five day storage in a space enviromment.

Figures $2 a$ and $2 b$ derived from Carton ( $\left.{ }^{1}\right)$ show the manner in which the selected operating conditions aflect the available payload. Incidentally, a more recent paper by Wrobel and Breshears ( ${ }^{3}$ ) has presented an analytical method which — for a given set of scaling constants - gives the initial acceleration which produces a maximum payload. 


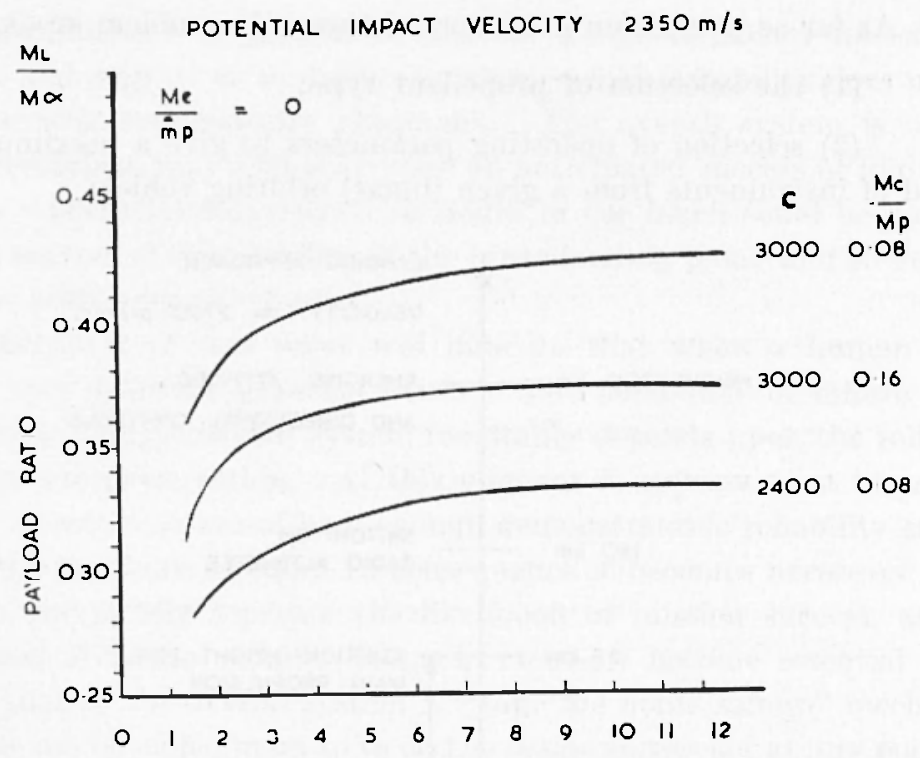

MaXIMUM aBSOLUTE aCCELERATION EARTH g's

Fig. $2 a$ - Vortical descent effect of scaling parameters.

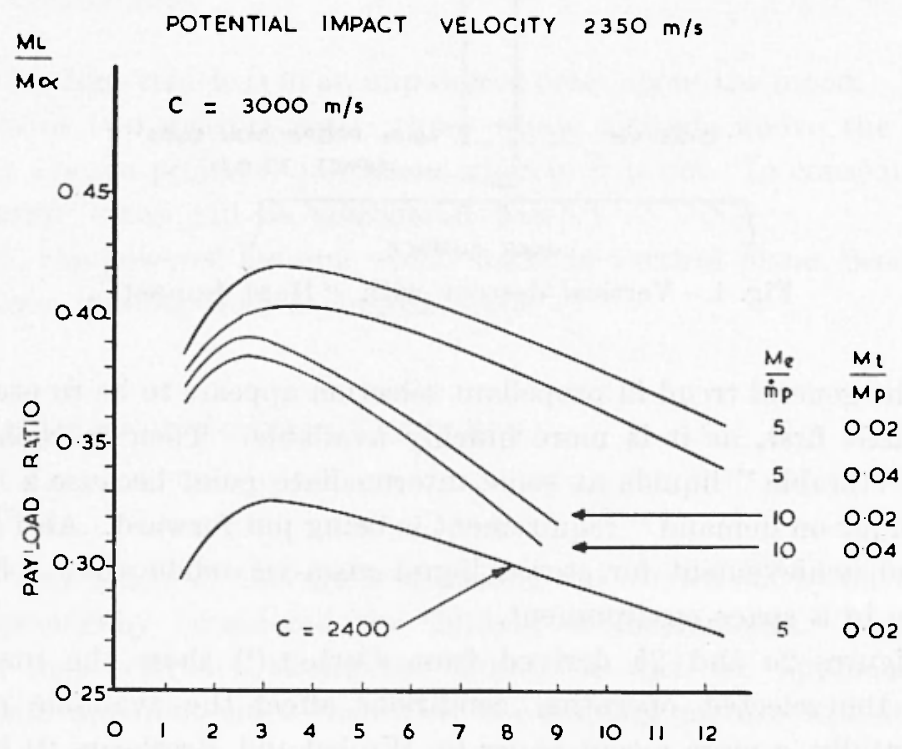

MAXIMUM ABSOLIITE ACCELERATION EARTH 9 's

Fig. $2 b$ - Vertical descent effect of scaling parameters. 


\section{Manned Vertical Descent ?}

A number of points may be raised in favour of carrying out vertical descent manned landings. This method achieves greater simplicity with respect to guidance and control and also reduces propulsion problems. In addition the possibility of having only one landing technique from early instrumentation soft landings (as for example Surveyor) through the manned programme is a very valid " good point".

There are unfortunately two serious drawbacks which make manned vertical descent most unlikely. The first of these is the very limited area of the lunar surface upon which it is possible to achieve " near vertical descent". However, the over-riding problems occur in the need to minimise the mass penalties in meeting safety requirements. It would appear that the slightly delayed start of a propulsion system, or operation at a thrust level below that planned could place a most severe load upon an escape capsule. Also, to do its job this capsule propulsion system would have to be of considerable proportions.

\section{DESCENT FROM ORBIT.}

Figure 3 shows the manner in which a distant non-circular orbit is converted to close circular by Hohmann transfer. Although the scale is

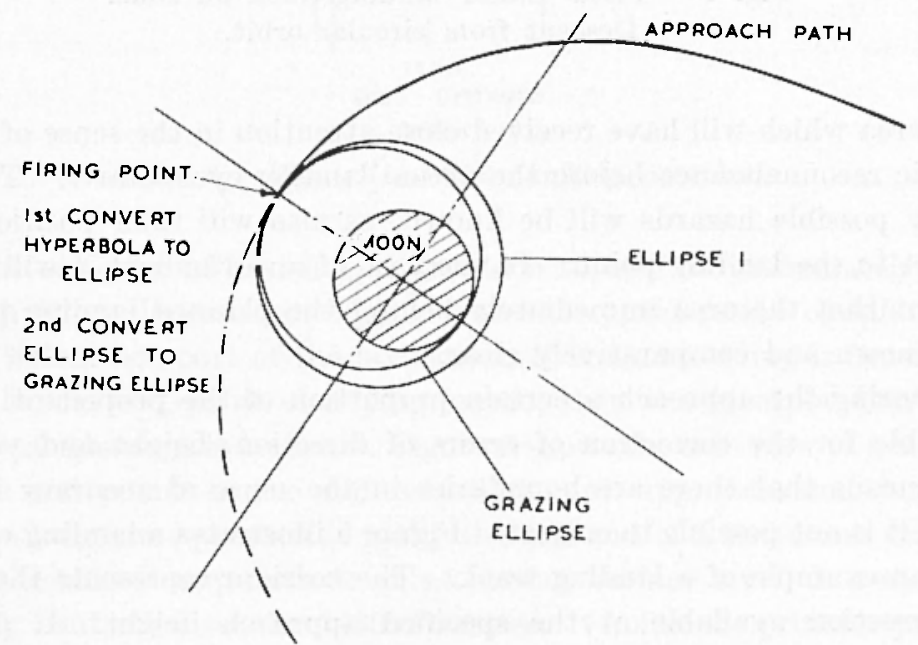

Fig. 3 - Lunar landing yperbolic-elliptic approach. 
considerably smaller than earth, analytically it is usually still possible to deal with the propulsive phase as though it were an impulse. This ceases to be true when the burning time represents a significant part of the orbit time.

It is assumed that the transfer will nake good most of the errors which have arisen (though most of these will have been corrected during the mid-course process). The landing vehicle is then in a close circular orbit passing very near to the planned landing point. (Figure 4). This

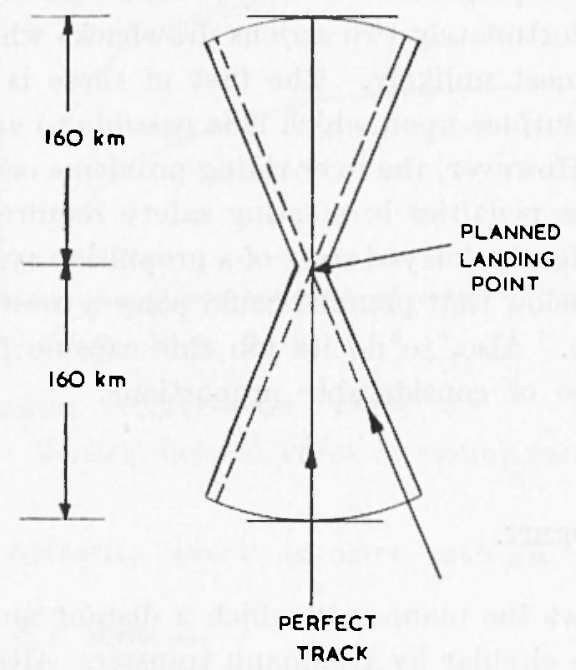

Fig. 4 - Plan. Iunar landing/track off zone. Descent from circular orbit.

is an area which will have received close attention in the sense of photographic reconnaissance before the actual landing cycle starts. The size of any possible hazards will be known, as also will their position with respect to the landing point. In the case of an overshoot it will be important that the area immediately behind the planned landing point is also known and comparatively clear.

During the approach a certain proportion of the propellant will be available for the correction of errors of direction, height and velocity. This means that there are boundaries in the sense of accuracy beyond which it is not possible to correct. Figure 5 illustrates a landing corridor with an example of a landing track. The corridor represents the limits of correction available at the specified approach height. It appears certain that the initial phase of guidance will be inertial because the 
landing point will not be visible using either light or radio. In consequence the relative data will be fed into the inertial system one revolution before touch down is attempted. If a fully automatic system is considered, then some form of position marker must be landed close to the planned landing point.

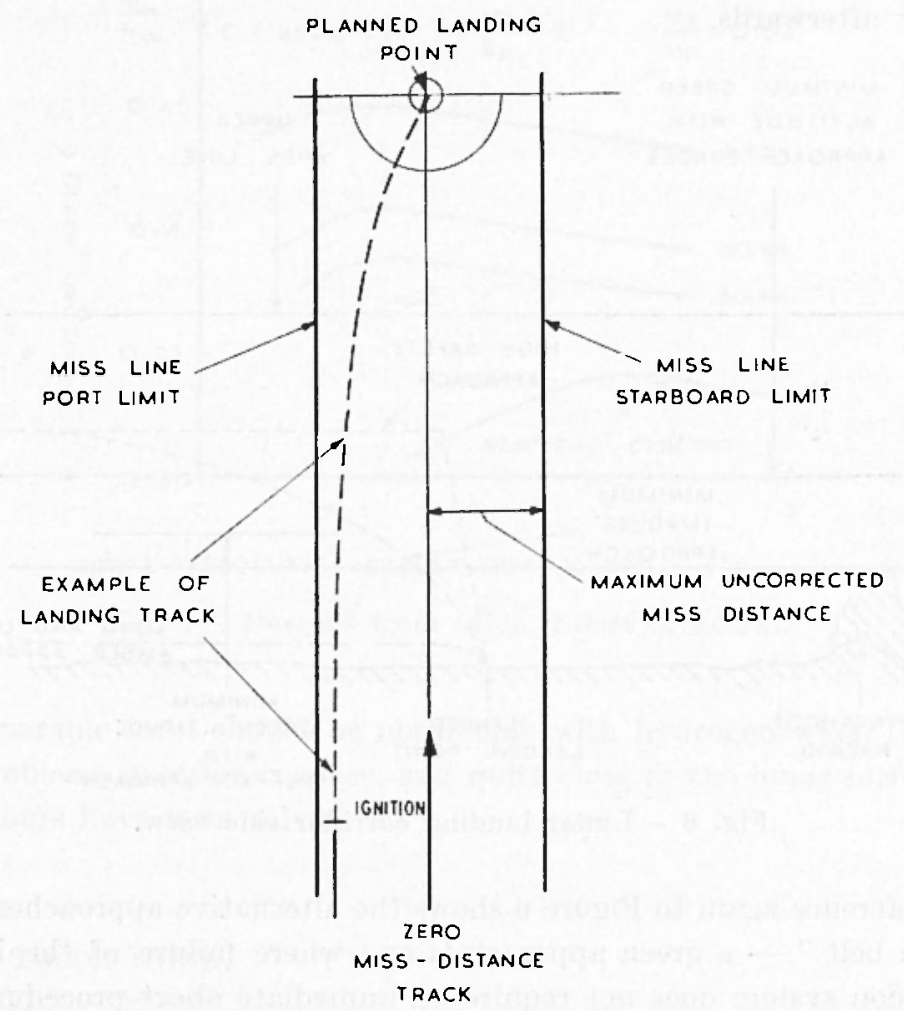

Fig. 5 - Plan of a lunar landing corridor.

The selection of best height at which to operate immediately prior to touch down depends considerably on the consideration of safety. If human beings are part of the layload, or if human landing is considered as the logical end point of a programme, then the incorporation of an "abort" mechanism is essential. Figure 6 shows the side view of a landing corridor and points to the different types of approach and landing.

The long low approach is really the tail end of a Hohmann transfer where the closest approach to m.s.l. (mean surface level) is very very carefully controlled to about 3000 metres. Choice of optimum propulsion arrangements can result in this approach giving a larger landed mass 
on the surface than any others considered. The gravity loss is reduced to insignificance. The main difficulties with this arrangement are due to the very high guidance accuracy required in the last part of the Hohmann transfer. Also from the safety point of view the failure of the landing propulsion system will result in a demand for an abort procedure very quickly afterwards.

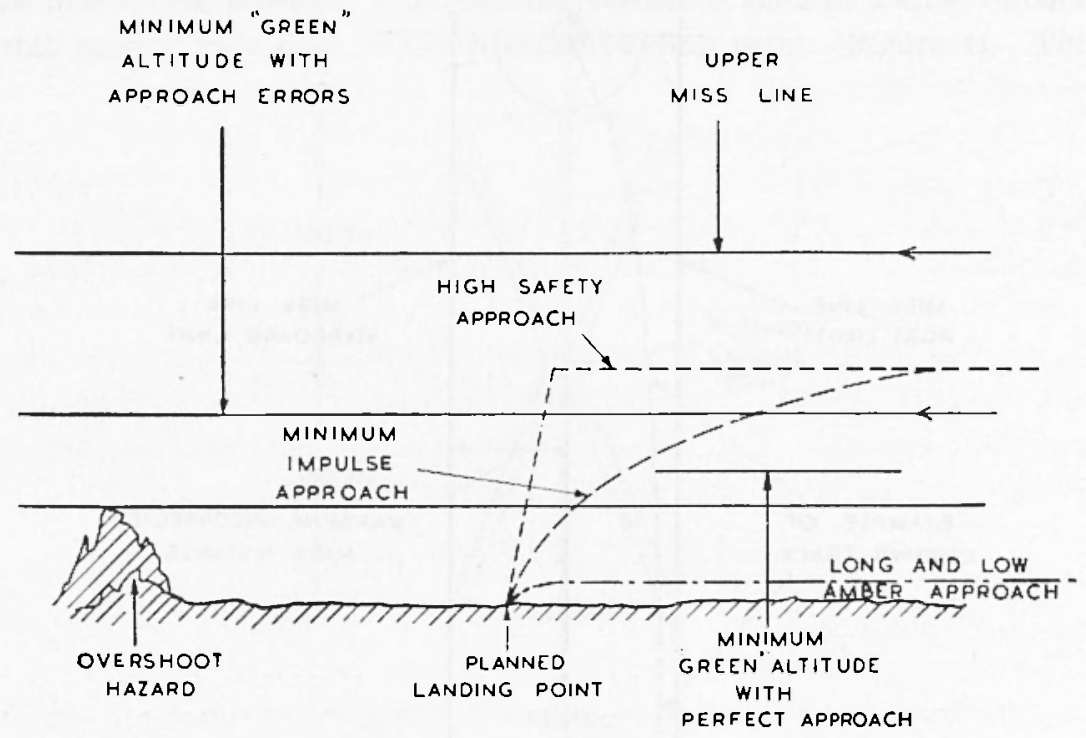

Fig. 6 - Lunar landing corridor side view.

Reference again to Figure 6 shows the alternative approaches in the "green belt" - a green approach is one where failure of the landing propulsion system does not require an immediate abort procedure. All parts of the trajectory are clear of the surface and also make allowance for altitude errors.

The trajectory "minimum impulse approach" represents the optimum transfer from whatever is the actual height and pass velocity to stationary at the landing point taking cognizance of the initial thrust available and the consequent acceleration. ( $\left.{ }^{4}\right)$. The problem of guidance of the vehicle down such an optimum descent regardless of possible variation of height and pass velocity, and possible propulsion disdispersion appears most difficult. In fact a number of modified approximations of this approach which are much less demanding in the guidance sense, and do not consume large excess quantities of propellant appear to be available. 
Figure 7 shows how sensitive the payload ratio is to the height of approach - based on a non — optimum but achievable descent programme. This is based in a liquid propellant engine using a comparatively straight-forward storable propellant combination. Better results, but

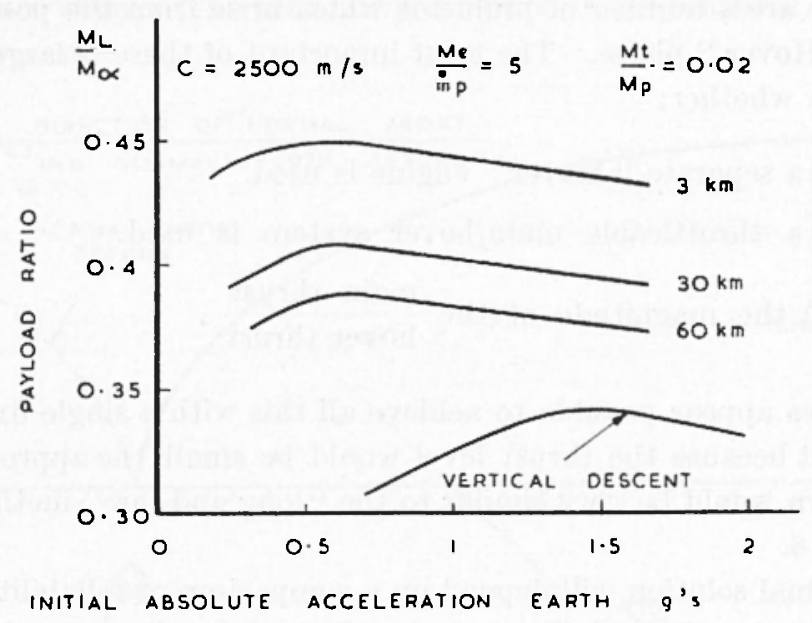

Fig. 7 - Descent from orbit. Effect of height.

of comparable form should be obtainable with hydrogen when the storage problems in cislunar space, and quite close to the lunar surface for some hours have been solved.

\section{LUNAR TOUCH DOWN.}

Two problems remained to be discussed - the effect of the propulsion system upon the lunar surface during the fint touch down, and the question of hovering. Not enough is known of the lunar surface for precise statements, but the possibility of disturbing large quantities of dust or shale may be serious. Apart from the problem of keeping visual contact with the planned landing point, the erosive properties may effect both window and radar. Some final approach "wriggle " may be required to keep the disturbance clear of the landing vehicle and its target.

Once the vehicle has been brought near to the surface and close to rest, the final movements are in the form of hovering. Because at this moment the vehicle is comparatively free of propellants, and also because of the low gravitational acceleration it is now possible to main- 
tain the vehicle at just the hover — thrust equalising weight — without excessive propellant demand. In fact a one mimure hover requires about $3 \%$ extra propellant. This should be long enough to make the final touch doxn very close to the position required.

There are a number of problems which arise from the possible need for the "Hover" phase. The most important of these is largely dependent upon whether:

(1) a separate "Hover" engine is used.

(2) a throttleable main/hover system is used.

(2b) the magnitude of the $\frac{\text { main thrust }}{\text { hover thrust }}$.

It does appear possible to achieve all this with a single fixed thrust engine, but because the thrust level would be small, the approach to the touch down would be very similar to the "long and low" method shown in Figure 6.

The final solution will depend on a comparison of reliabilities of two engines - of a throttleable engine and the hazards of the low thrust down the valley approach.

\section{THE ABORT CAPABILITY.}

During the lunar landing phase it is necessary that an abort capability be available in the landing velicele. This means that the human payload should occupy a part of the earth return and re-entry vehicle. In the event of a red failure the abort mechanism should safely discard the lunar landing section and place the remainder on a double green trajectory for home.

A most important aspect will be the minimising of the propulsion requirement in terms of total impulse, and also to make a single abort mechanism serve at all points to lunar touch down.

Referring to Figure 8, this shows the situation which will exist at various times to touch down from a circular orbit. Obviously a failure of the switching mechanism and consequent failure of the landing propulsion without ignition is not a severe problem. The vehicle is alleady on a green trajectory. The lunar landing section is jettisoned and only a small impulse is then required from the normal lumarearth return stage to place them on a green-green trajectory. 
If at the end of the landing phase in the final reetical descent, but at some time before touch down, the main lunar landing propulsion system fails, the abort requilement is one of fast response. The velocity increment is a very small amount greater than the normal lumar surfaceearth return stage. The turn down direction is not sensitive at this encl of the proeess.

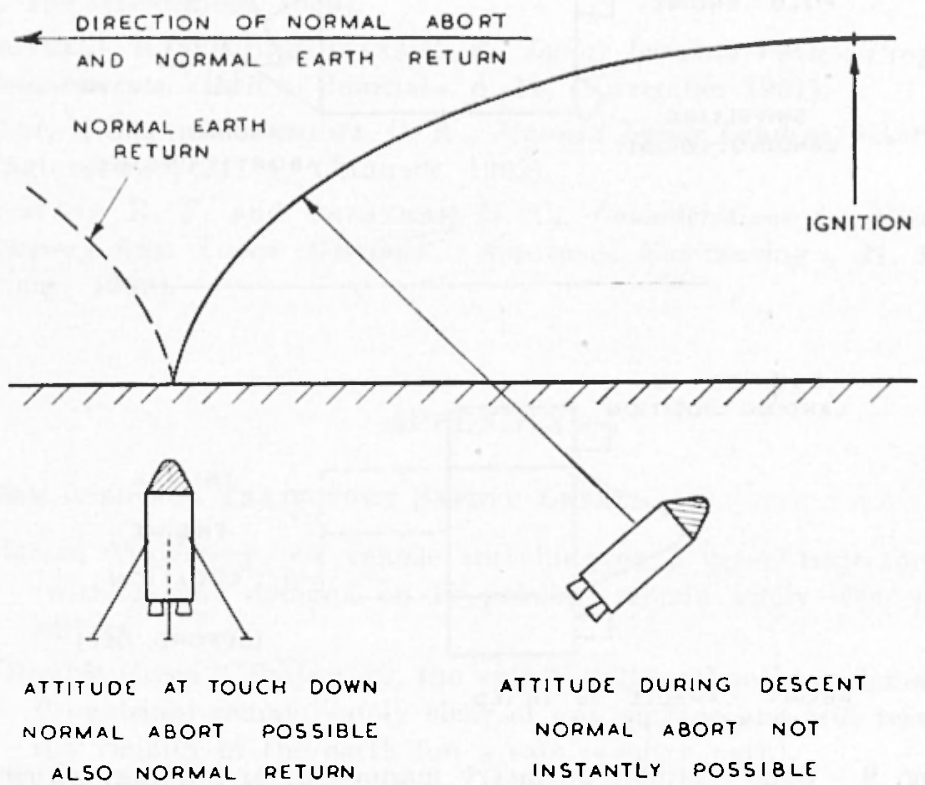

Fig. 8 - Lunar landing with fixerl greometry vehicles.

At any point intermediate between ignition failure and propulsion failure in final descent the problem oceurs like this.

Following a successful ignition, rehicle relocity has been brought below circular. Trajectory angle dips out of horizontal. Consider as an eximple an angle of $45^{\circ}$, an instantaneous relocity of $1000 \mathrm{~m} / \mathrm{s}$. At this moment the propulsion system fails. The velocity increment required by the abort vehicele if it is to retum back in the direction it has just come, is apploximately circular plus $1000 \mathrm{~m} / \mathrm{s}$, say a maximum requirement in this manner of $2750 \mathrm{~m} / \mathrm{s}$. which would have to be avalable in the normal lunar surface-earth return stage. On the other hand, if it were possible for the abort machine to get chear of the crashing landing stage and turn to the best departure angle it would only need a velocity incement of say $1000 \mathrm{~m}$ s less than the normal requirement 
of the earth return stage. Figure 8 shows up the problems in trying to maintain an abort mechanism in a fixed geometry velicele through the landing phase.

A possible alternative showing some variable geometry landing vehicles may in fact ofler a number of attractive facets (Fig. 9).
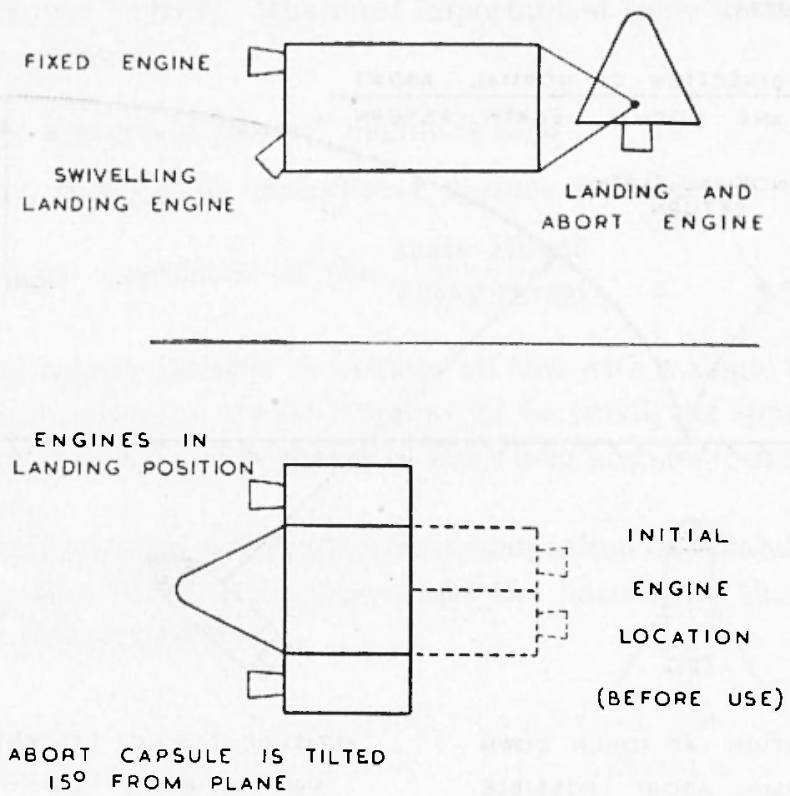

Fig. 9 - Some variable geometry mamed lunar landing vehicles.

The first of these could have the rotating capsule in landing position, and with the engine going before the main propulsion system brought the vehicle into land. At touch down both swivelling engines would work as hover engines.

The second layout would permit a clear view of proceedings to be made throughout the landing phase plus a free way to a minimum energy abort mode.

Note. - The study, of which this paper forms a part, was largely com. plete before the author became aware of the "Man into Space" issue of the Institute of Aerospace Science publication "Aerospace Engineering". 'This compendinn contains the nost comprehensive and authoritative statement of what may be termed the "NASA mannerl lunar lanrling philosophy". Iu particular, reference is made to the work of Faget and Mathews (4) and Thompson and Cheatham $\left.{ }^{5}\right)$ whose work overlaps the present paper at a number of points. 


\section{REFERENCES}

(1) Cartox, D. S., Minimum Propulsion for soft-moon-landing of instruments. "College of Acronautics ", Note 9 4 , July 1959, Cranfield, England and in "Spaceflight Technology", Ed. K. W. Gatland, Academic Press, (1960).

$\left({ }^{2}\right)$ Carton, D. S., Landing Instruments on the Moon. "The New Scientist", 6, 159, (Decenber 1959).

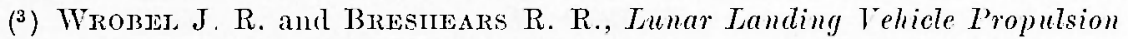
Requirements. "A.R.S. Journal ", 6, 11, (November 1961).

(4) FAciet, M. A. and MATHEws, C. W., Manned Lunar Landing. "Aerospace Engineering ", 21, 1, (January 1962).

$\left({ }^{5}\right)$ Thompson R. F. and Cimbatiun 1). C., Considerations for Spacecraft Recivery from Lunar Missions. "Aerospace Engineering", 21, 1. (January, 1962).

\section{APPENDIX}

Notation Usjed for Trajectory safety Ljevej.

1. - "Green Trajectory. A vehicle travelling on a green trajectory will (without any demand on Propulsion) remain safely clear of any surface.

2. - "Double Green" Trajectory, the vehicle will (without any demand on Propulsion) remain safely clear of any surface and will return to the vicinity of the earth (on a safe re-entry path).

3. - "Red" Trajectory. The vehicle is under propulsion or about to be propelled. The failure of the propulsion system will require in. mediate abort action.

4. - "Amber" Trajectory, the vehicle is under propulsion. The failure of the propulsion system will require abort action.

5. - "Yellow" Trajectory, the vehicle is not under propulsion. Without the action of the propulsion system the velicle will not remain safely clear of any surface. 